\title{
Iterative reconstruction optimisations for high angle cone-beam micro-CT
}

\author{
B. Recur, M. Fauconneau, A. Kingston, G. Myers and A. Sheppard \\ Dept. Applied Mathematics, RSPE, Australian National University, Canberra, AUSTRALIA
}

\begin{abstract}
We address several acquisition questions that have arisen for the high cone-angle helical-scanning micro-CT facility developed at the Australian National University. These challenges are generally known in medical and industrial cone-beam scanners but can be neglected in these systems. For our large datasets, with more than $2048^{3}$ voxels, minimising the number of operations (or iterations) is crucial. Large cone-angles enable high signal-to-noise ratio imaging and a large helical pitch to be used. This introduces two challenges: (i) non-uniform resolution throughout the reconstruction, (ii) over-scan beyond the region-of-interest significantly increases required reconstructed volume size. Challenge (i) can be addressed by using a double-helix or lower pitch helix but both solutions slow down iterations. Challenge (ii) can also be improved by using a lower pitch helix but results in more projections slowing down iterations. This may be overcome using less projections per revolution but leads to more iterations required. Here we assume a given total time for acquisition and a given reconstruction technique (SART) and seek to identify the optimal trajectory and number of projections per revolution in order to produce the best tomogram, minimise reconstruction time required, and minimise memory requirements.
\end{abstract}

Keywords: X-Ray Tomography, Iterative Reconstructions, High cone-angle beam.

\section{INTRODUCTION}

The micro-CT facility developed at ANU uses high cone-angle helical-scanning systems. The high cone-angle is achieved through a large 2D flat-panel detector (400x300 mm, 2048x1536 pixels) positioned $300 \mathrm{~mm}$ from the X-ray source. This allows us to capture a large solid angle of the X-ray flux being emitted from the X-ray source with a spherial wavefront leading to very high signal-to-noise-ratio (SNR) imaging. Using a large number of projections per revolution, e.g., 2520, the well-known Katsevich "theoretically exact" algorithm ${ }^{1}$ is able to reconstruct the tomogram of the scanned object regardless of cone-angle. However, with this geometry, we encounter several challenges:

(i) in order to image specimens at 1 micron resolution requires they be positioned $1.5 \mathrm{~mm}$ from the source, resulting in an average magnification of 200x. However, magnification varies by a factor of 5 across the specimen. The large change in magnification leads to a non-uniform resolution of the reconstructed data; The reconstructed regions close to the source trajectory are sharp and those far away from the source trajectory are blurry. We have shown that a "double helix" scan composed of two symmetric helices can solve this problem. ${ }^{2,3}$ This has the advantage of reducing the non-uniform resolution by combining both volumes obtained from each single-helix reconstruction, however, it increases both the acquisition and reconstruction time.

(ii) Katsevich filtering requires data to lie on $\pi$-lines, so voxels at the extremities of the reconstructed volume require an additional half rotation of scanning beyond the edge of the sample. This is termed the "overscan". A full rotation of overscan is required per helix.

(iii) The amount of data required for Katsevich reconstruction from a double helix trajectory is quite significant and is not optimally used for a number of reasons: a) A large number of projections per revolution are required for faithful inter-projection filtering, b) two full rotations of overscan are included, and c) less than $50 \%$ of the data in each projection lies within the Tam-Danielsson window and is thus back-projected.

Iterative reconstruction techniques such as the Simulatenous Algebraic Reconstruction Technique (SART) ${ }^{4-6}$ or the Expectation-Maximization for TRansmission tomography (ML-TR) ${ }^{7-9}$ are good candidates to provide high

Further author information (Send correspondence to B. Recur): benoit.recur@anu.edu.au

Developments in X-Ray Tomography IX, edited by Stuart R. Stock, Proc. of SPIE Vol. 9212, $921214 \cdot$ (c) 2014 SPIE · CCC code: 0277-786X/14/\$18 · doi: 10.1117/12.2062450 
quality reconstruction. They can make full utilisation of the data in each projection and can deal with arbitrary, discontinuous, or even random source trajectories and a limited number of projections. They also enables us to incorporate iterative correction methods since they can simulate many experimental effects of the acquisition in the forward model and compensate for them. For instance, they can deal with projection misalignments observer on the detector due to the source movements and translation/rotation stage positioning errors. ${ }^{10,11}$ They can also simulate the non-linear effects of a polychromatic source in order to correct for beam hardening artefacts. ${ }^{12}$ In companion papers, we use iterative techniques to perform simultaneous reconstruction/material classification from a dual-energy scans ${ }^{13}$ and capture time-resolved continuous fluid flow in dynamic tomography. ${ }^{14}$

With this in mind, here we are assuming an iterative reconstruction and, in order to improve the overall work-flow developed at the ANU, we are are seeking the optimal experimental acquistion protocol in order to produce the best tomogram with minimal reconstruction time required and minimal memory requirements. In particular we are looking for an acquisition which is able to reduce non-uniform high magnification effects with minimal overscan issues enabling the best use of the data and a fast reconstruction. The remainder of the paper is organised as follows: In section 2, we describe the different investigated acquisition configurations, introduce the simulation study protocol and detail the iterative reconstruction which has been used in this study. Then, reconstruction results are discussed in section 3 to determine the best configuration candidates for our high-cone angle imaging system.

\section{NUMERICAL ANALYSIS MATERIAL AND METHODS}

In this section we first detail the numerical ground truth phantom and simulated acquisition protocol. Then we develop the different acquisition parameters (pitch, projection number, aperture time) we deal with in order to define the best acquisition scheme according to different source trajectories. We finally introduce the iterative reconstruction technique used to compute the tomographic results discussed in this paper.

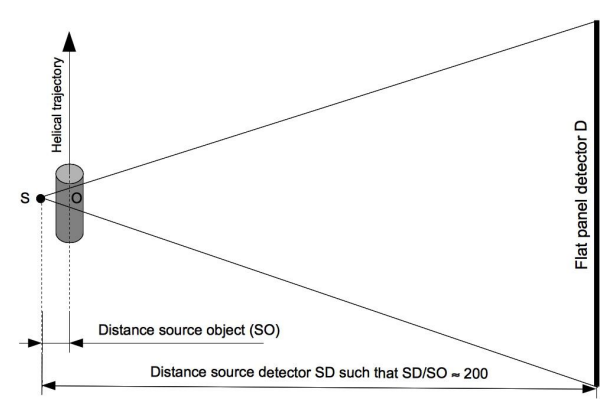

(a)

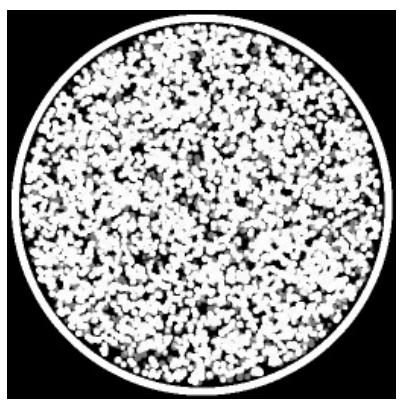

(b)

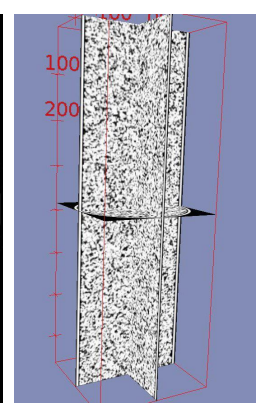

(c)

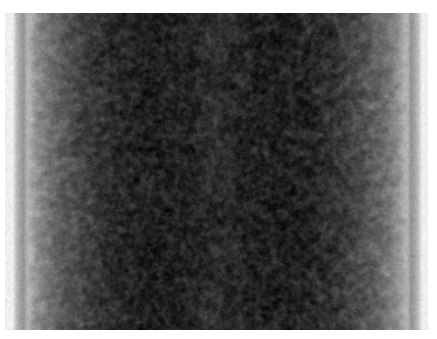

(d)

Figure 1. (a) Scanner geometry. (b) Central slice and (c) 3D rendering of the simulated rock sample rasterized into a $256^{2} \times 768$ voxel volumes. (d) Example of projection computed from the sample.

\subsection{Analytic acquisition of simulated object}

This study is based on a numerical analysis. A synthetic volume simulating a rock sample is computed and used as ground truth. This sample is composed of a container filled randomly with different mineral grains. The container is a $6 \mathrm{~mm}$ diameter cylinder shell in aluminium. Each grain is modelled as a sphere with a radius chosen uniformly between $100 \mu \mathrm{m}$ and $250 \mu \mathrm{m}$. The mineral composition we have chosen is $60 \%$ of quartz, $20 \%$ kaolinite and $20 \%$ calcite. Tab. 1 summarizes densities, attenuation values and quantity of each material composing the sample.

The phantom (or synthetic object) is acquired at higher resolution using a detector with twice the number of pixels in each dimension to prevent the inverse crime. ${ }^{15} \mathrm{X}$-ray paths are traced from source to each pixel of the detector and the distance crossed by each X-ray path through each material composing the object is measured and total absorption is computed for each sub-pixel $i i$ by $A(i i)=\sum_{m \in M_{s}} \int \mu_{m}(x) d x$, where $M_{s}$ is the material 


\begin{tabular}{|c||c|c|c|c|c|}
\hline Material $m$ & Air & Aluminium & Kaolinite & Quartz & Calcite \\
\hline Quantity & & & $20 \%$ & $60 \%$ & $20 \%$ \\
Attenuation & 0.01 & 30.38 & 29.25 & 29.82 & 30.49 \\
Density & 0.001 & 2.7 & 1.3 & 2.6 & 2.6 \\
\hline
\end{tabular}

Table 1. Composition of the numerical rock sample (in \% of sample volume), attenuation $\left(\mathrm{m}^{-1}\right)$ and density $\left(\mathrm{g} . \mathrm{cm}^{-3}\right)$.

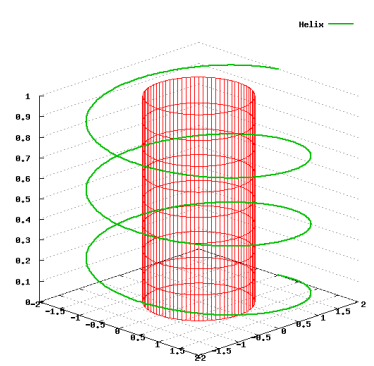

(a)

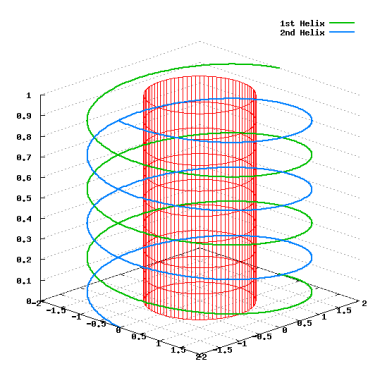

(b)

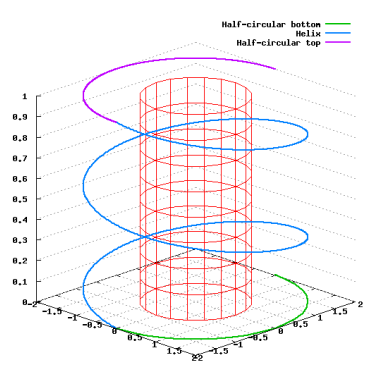

(c)

Figure 2. Three investigated source trajectories: (a) standard single-helix, (b) double symmetric helices, (c) adaptive trajectory composed of 2 circular scans at the edge of the object of interest and a single-helical trajectory between them.

set composing the sample and $\mu_{m}$ corresponds is the attenuation for material $m$ (cf. Tab. 1). We obtain the final projection value on the detector by averaging the four sub-pixel values and applying a Poisson noise model:

$$
R(i)=\text { Poisson }\left\{\sum_{i i \in i} \gamma_{0} \exp (-A(i i))\right\},
$$

where $\gamma_{0}$ is the average photon counts on detector (blank scan) and $i i \in i$ denotes sub-pixel indexes contained in the detector pixel $i$.

We have also rasterised the analytic object into an $N^{2} \times M$ voxel volume for comparison with reconstruction results, (i.e. $N^{2} \times M$ also corresponds to reconstructed volume size: $M$ slices of $N^{2}$ pixels). Edges are approximated by linear interpolation. As an illustration, Fig. 1(b-c) respectively show the $2 \mathrm{D}$ central slice and 3D multi-planar rendering of the ground truth volume. Fig. 1(d) is a linearized projection computed from the simulated object (linearisation performed for better visualisation). In this study, $N^{2} \times M=256^{2} \times 768$ voxels and detector size is $256 \times 192$ pixels.

\subsection{Source trajectories}

Three source trajectories are investigated (c.f. Fig. 2). The first one consists of a single-helix using an uniform vertical pitch. This configuration corresponds to the standard helical scan performed on our system that maximises the Tam-Danielson window.

The second trajectory consists of a double-helix scan. A first helix is acquired, then the sample is rotated by $\pi$ and a second helix (symmetric to the first one) is performed. Currently, a tomogram is computed for each single-helix using the Katsevich algorithm and both are merged to provide final reconstruction result. For a high cone-angle helical scanner, it has been demonstrated ${ }^{2,3}$ that double-helix acquisitions reduce deformations and non-uniform resolution due to the high magnification, especially at the edges of the sample.

The third investigated trajectory, denoted "adaptive trajectory," performs half circle trajectories at the top and bottom of a helical scan in place of the overscans. This should minimise the aditional reconstruction volume required in iteration to perform forward projection. 

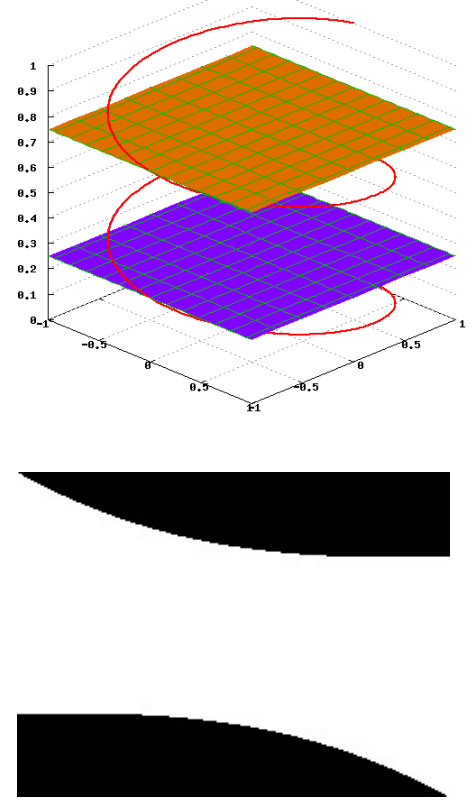

(a)
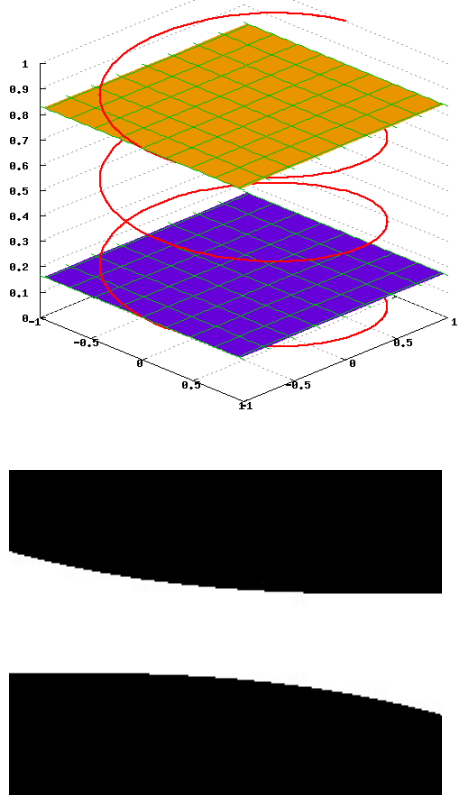

(b)
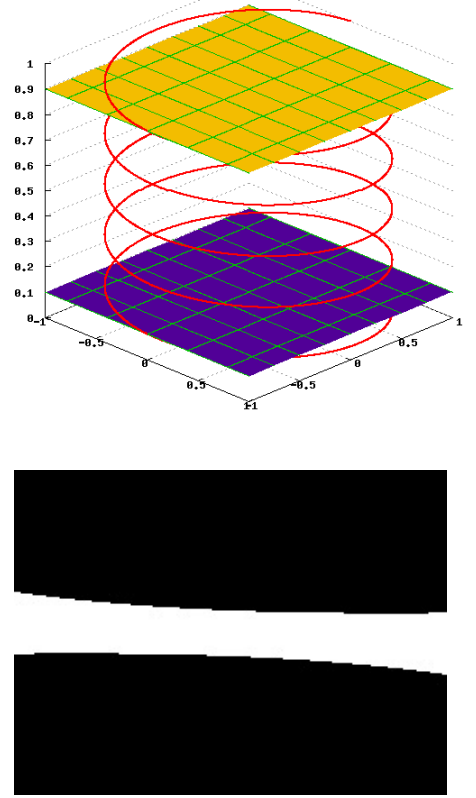

(c)

Figure 3. (a) Optimal pitch and associated Tam-Danielson Window (black area of the picture corresponds to the redundant data). (b) Half pitch (redundancy $\times 2$ ) (c) Quarter pitch (redundancy $\times 4$ ). Volume between top and bottom planes on the pitch trajectories delimit the complete reconstruction region. Outer unwanted volumes, have to be computed to achieve the reconstruction correctly.

\subsection{Acquisition parameters}

The parameters we deal with are the vertical pitch $P$, the number of projections per revolution, $N_{\theta}$, (note that this affects the average number of counted photons in blank scan $\gamma_{0}$ ), and the number of revolutions $N_{r}$. We denote the relative pitch $P=1$ as the pitch which maximizes the Tam-Danielson window, i.e., that which minimizes data redundancy between two consecutive revolutions. This configuration allows us to perform a fastscan since redundancy is minimal. Conversely, it leads to a non-uniform resolution with a high-cone angle helical scanner. Thus we reduce relative pitch to 0.5 and $0.25(\times 2$ and $\times 4$ redundancy on projections $)$ to investigate quality limitation due to high-pitch when all the other parameters are optimal. The considered pitches are presented in Fig. 3: trajectories are drawn in red curves. The top and bottom planes delimit the region correctly reconstructed (between them) whereas the outer parts have to be reconstructed as well to achieve a correct reconstruction of the object. Reducing the pitch leads to a reduction in the additional reconstructed "buffer" volume size required.

We also investigate the effect of a smaller/larger projection number with respect to the relative pitch for each trajectory. Let $N_{\theta}=\frac{\pi}{2} N$ the approximate projection number per revolution to meet Nyquist sampling criterion in a standard single-helix scan, (i.e., $N_{\theta}=400$ in our study). Similar to pitch, we measure the quality effects with $N_{\theta}, \frac{N_{\theta}}{2}$, and $\frac{N_{\theta}}{4}$.

Finally, since one of our goals is to the best result for a given acquisition time, we modify the aperture time simulate all experimetns of the same duration. Let $\gamma_{0}=10000$ be the average number of photons in standard $\operatorname{scan}\left(N_{\theta}=400\right.$ and $\left.P=1\right)$. For each acquisition protocol investigated, we reduce the aperture time by reducing $\gamma_{0}$ to ensure the overall acquisition time remains constant according to the total number of projecions, $N_{\theta} \times N_{r}$. A summary of the source trajectory and acquisition parameter configurations investigated is included in Tab. 2. As an example, Fig. 4 shows the same projection obtained by the single-helix configuration $\left(N_{\theta}=100, N_{r}=5, \gamma_{0}=16000\right)$ (a), and the double-helix configuration $\left(N_{\theta}=400, N_{r}=10, \gamma_{0}=2000\right)$. Fig. 4(c) 


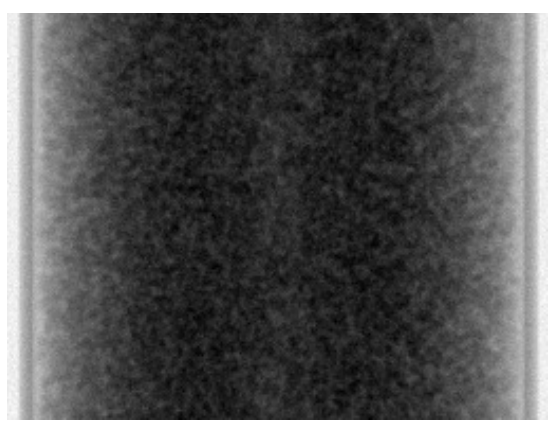

(a)

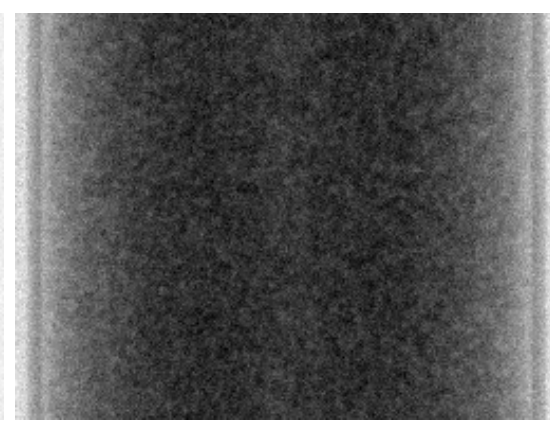

(b)

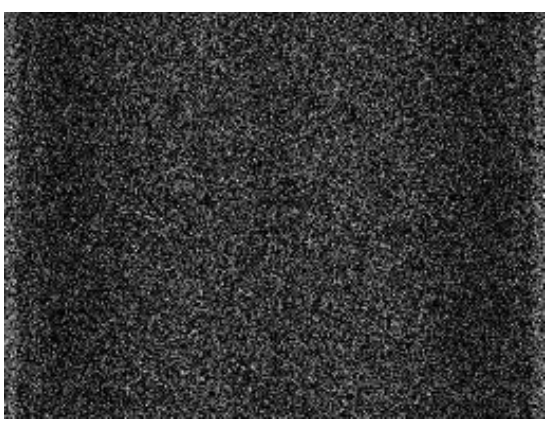

(c)

Figure 4. (a) Projection of configuration $\left(N_{\theta}=100, N_{r}=5, \gamma_{0}=16000\right)$, single trajectory. (b) Projection of configuration $\left(N_{\theta}=400, N_{r}=10, \gamma_{0}=2000\right)$, double-helix. (c) Difference highlighting the amount of noise, mainly in (b), compared to (a) (scale has been set to $0.25 \times$ the scale of (a) and (b) for better visualisation).

\begin{tabular}{|c|c|c|c|}
\hline$N_{\theta}$ & $N_{r}$ & $P$ & $\gamma_{0}$ \\
\hline 400 & 2 & 1 & 10000 \\
400 & 3 & 2 & 6667 \\
400 & 5 & 4 & 4000 \\
\hline 200 & 2 & 1 & 20000 \\
200 & 3 & 2 & 13334 \\
200 & 5 & 4 & 8000 \\
\hline 100 & 2 & 1 & 40000 \\
100 & 3 & 2 & 26667 \\
100 & 5 & 4 & 16000 \\
\hline
\end{tabular}

(a)

\begin{tabular}{|c|c|c|c|}
\hline$N_{\theta}$ & $N_{r}$ & $P$ & $\gamma_{0}$ \\
\hline 400 & 4 & 1 & 5000 \\
400 & 6 & 2 & 3333 \\
400 & 10 & 4 & 2000 \\
\hline 200 & 4 & 1 & 10000 \\
200 & 6 & 2 & 6667 \\
200 & 10 & 4 & 4000 \\
\hline 100 & 4 & 1 & 20000 \\
100 & 6 & 2 & 13333 \\
100 & 10 & 4 & 8000 \\
\hline
\end{tabular}

(b)

Table 2. (a) Parameters of the acquisition for: (a) the single-helix and adaptive trajectories, and (b) double-helix trajectory.

highlights the noise (mainly given from (b)) between the very high-quality projection (a) and the worst quality projection (b).

\subsection{Reconstruction method}

Several iterative reconstruction methods have been proposed over the last few decades, such as conjugate gradient, algebraic reconstruction techniques, ${ }^{4-6}$ and expectation maximization algorithms for emission and transmission tomography. ${ }^{7,8}$ Since this paper focuses on the acquisition scheme optimisations, we have based our discussion on images reconstructed with the ordered subsets convex (OSC) algorithm which implements the expectation maximization for transmission tomography. ${ }^{9}$

OSC consists of iterating in $t$ and subsets, $s+1$, in order to update each voxel, $j$, of the volume, $f$, representing the acquired object, until convergence is achieved. The volume obtained at sub-iteration $s$ is used as starting volume for the next sub-iteration $s+1$. The algorithm updates each voxel as follows:

$$
f_{s+1}^{t}(j)=f_{s}^{t}(j)+\frac{\sum_{i \in S(s)} w_{i j}\left(\hat{R}_{s}^{t}(i)-R(i)\right)}{\sum_{i \in S(s)} w_{i j}\left[\sum_{k} w_{i k}\right] \hat{R}_{s}^{t}(i)}
$$

where $\hat{R}_{s}^{t}(i)$ is the expected photon counts computed from $f_{s}^{t}, S(s)$ are the radiographs in the subset $s . \hat{R}$ is modeled by:

$$
\hat{R}(i)=\gamma_{0}(i) \mathrm{e}^{-\sum_{j} w_{i j} f(j)}+b g(i)
$$




\begin{tabular}{|c|c|c|c|c|c|c|c|c|c|c|c|c|}
\hline$N_{\theta} \times N_{r}$ & 200 & 300 & 400 & 500 & 600 & 800 & 1000 & 1200 & 1600 & 2000 & 2400 & 4000 \\
\hline$\#$ Iter $_{\max }$ & 40 & 27 & 20 & 16 & 13 & 10 & 8 & 7 & 5 & 4 & 3 & 2 \\
\hline$\# R-R^{-1}$ & 8000 & 8100 & 8000 & 8000 & 7800 & 8000 & 8000 & 8400 & 8000 & 8000 & 7200 & 8000 \\
\hline
\end{tabular}

Table 3. According with the configurations in Tab. 2, the overall projection number on an acquisition is $N_{\theta} \times N_{r}$. By limiting the iteration number \# Iter $_{\max }$, we assume an almost similar computation time, proportional to \# $R-R^{-1}$, for all reconstructions.

where $\gamma_{0}$ and $b g$ are blank calibration scan and dark field, respectively, and $w_{i j}$ is a weight coefficient defining the contribution of voxel, $j$, to detector pixel, $i$. A main iteration $t$ is completed when all subsets have been processed. Projection order has an influence on convergence to update the volume. It is known that the sequential order is not optimized because two successive projections are too correlated (i.e. the second one does not contain a large amount of unknown data compared to the first one). Thus in this paper, we have used the optimized multi-level scheme (MLS). ${ }^{16}$ It selects the next projection as the one which is the least correlated with the projections already used within an iteration $t$.

With respect to the acquisition time limit, we have forced the iterative algorithm to a reconstruction time limit, i.e. a maximum number of projection-backprojection processes (denoted $\# R-R^{-1}$ ), so that the maximum computation time remains the same (or almost the same) for each acquisition configuration. Computation limitations are summarised in Tab. 3. According to the scan configurations in Tab. 2, the overall projection

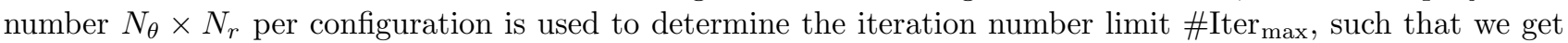
an almost similar computation time, proportional to $\# R-R^{-1}$, for all reconstructions. We have also added a convergence limit based on the quadratic residual error so that reconstruction is stopped if, i) the projection quadratic error $\sum_{i}\left[\hat{R}_{s}^{t}(i)-R(i)\right]^{2}$ is less than $5 \%$ of the initial backprojected error $\sum_{i}[R(i)]^{2}$, or if, ii) a maximum of \#Iter max iterations have been performed. We discuss the convergence of each scan configuration later when determining the most appropriate trade off between acquisition and reconstruction times and consider which criterion, i) or ii), has halted the computation.

\section{RESULTS AND DISCUSSION}

Simulated acquisitions obtained from our numerical sample (27 different configurations, cf. Tab. 2) are processed by the OSC algorithm. Reconstruction iterations have been stopped when one of the two criterion explained in previous section has been verified. Convergence curves corresponding to the overall residual error backprojected into the volume according to the number of operations $\left(\# R-R^{-1}\right)$ are given on Fig. 5. Despite the iteration limit \#Iter $\max$, one can remark that all reconstructions have globally reached the convergence position since the error becomes constant when the operation number increases. Moreover, some reconstructions have stopped for $\# R-R^{-1}<8000$, due to criteria i), especially when $N_{\theta}$ is low. However some configuration have not reached the best convergence solution, especially when $N_{\theta} \times N_{r}$ is high.

The central slice of the single-helix trajectory reconstruction results are shown in Fig. 6. First, consider the $N_{\theta}=400$ projections per revolution results. We notice that reconstructed images become noisier with a larger pitch. Although projections are $4 \times$ noisier when $P=0.25$ than when $P=1$ (since we have specified constant acquisition time), the averaging made in the each voxel when $P \leq 0.5$ provides sharper and less noisy solutions. Next, when reducing $N_{\theta}$ to 200 , we reduce the effect of noise in the reconstruction while still maintaining a reasonable sharpness. We see, for example, comparable results between $\left(N_{\theta}=400, P=0.5\right)$ and $\left(N_{\theta}=200, P=0.25\right)$. We can also observe that a number of projections per revolution that is too low yields blurry solutions, e.g., $P \geq 0.5$ when $N_{\theta}=100$. The similarly blurry result obtained for $\left(N_{\theta}=200, P=1\right)$, even though it converged, highlights an accuracy limitation due to the single-helix configuration.

Figure 9 gives difference images for $\left(N_{\theta}=400, P=0.5\right),\left(N_{\theta}=200, P=0.5\right)$ and $\left(N_{\theta}=100, P=0.5\right)$ with the original slice. In the error image in Fig. 9 (a) the edges have not been reconstructed due to a lack of data in the projections. The best results are obtained when the projection number satisfies Nyquist criterion (Fig. 9(c)), however, quality improvements compared to intermediate configuration $\left(N_{\theta}=200, P=2\right)$ are not significant due to the limited reconstruction time. 


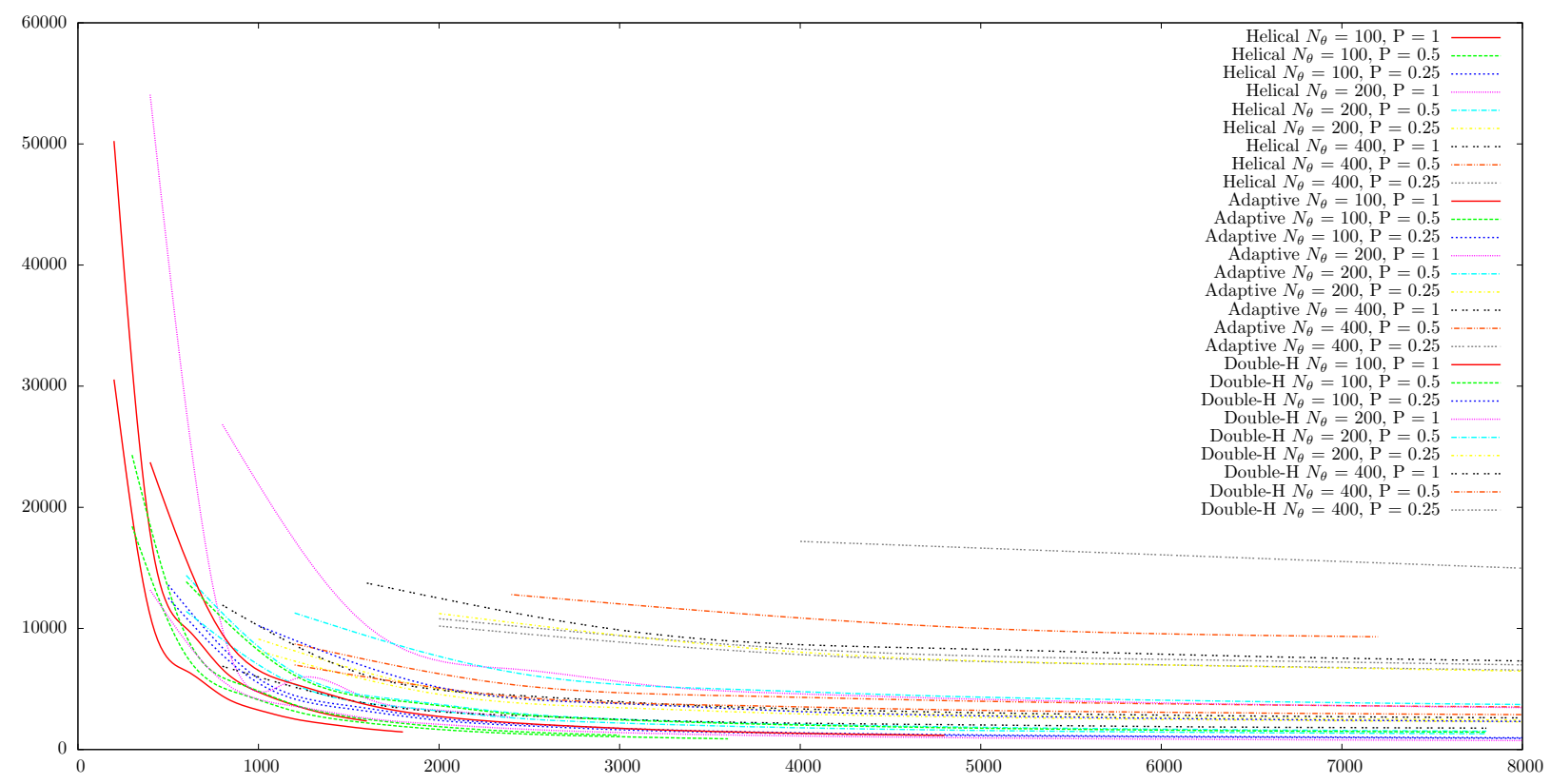

Figure 5. Convergence curves (residual error backprojected into the reconstructed volume) according to the number of operations $\# R-R^{-1}$.

A large $N_{\theta}$ leads to a sharp but noisy solution (e.g. $\left(N_{\theta}=400, P=1\right)$ ). The noise is reduced by using a lower pitch (e.g. $\left(N_{\theta}=400, P=0.5\right)$ or $\left(N_{\theta}=400, P=0.25\right)$ ), but at the cost of computation time assuming convergence without an iteration limit. An intermediate configuration also reaches a reasonable solution (sharp with low noise) with a better convergence rate, as observed for $\left(N_{\theta}=200, P=0.5\right),\left(N_{\theta}=200, P=0.25\right)$ or $\left(N_{\theta}=100, P=0.25\right)$. Finally, too low a total number $N_{\theta} \times N_{r}$ always achieves a blurry solution due to the non-uniform resolution issues of high-cone angle imaging.

The central slice of the double-helix trajectory reconstruction results are depicted in Fig. 7. Similar to the single-helix, noise effects are reduced with a lower pitch. However, results obtained for all $P$ when $N_{\theta}=400$ and $N_{\theta}=200$ are comparable, so a double-helix configuration also reduces noise effect when $P=1$ (even if noise is $\times 2$ in the projections) since each voxel value is averaged twice (by each helix) compared to the similar single-helix configuration. However, due to the inherently large number of projections $\left(N_{\theta} \times N_{r}\right)$, convergence is not reached at the iteration limit. A reasonable trade of between iterations and the given acquisition time, seems to be the diagonal $\left(N_{\theta}=400, P=1\right),\left(N_{\theta}=200, P=0.5,\left(N_{\theta}=100, P=0.25\right)\right.$.

Fig. 8 shows the the central slice of the reconstruction results for the adaptive trajectory. Again, we observe that, i) $N_{\theta}=400$ results suffer from the level of noise in the projections, and, ii) the noise effect is reduced by a lower pitch despite the higher noise in the acquisition. $\left(N_{\theta}=100, P=1\right)$ and $\left(N_{\theta}=100, P=2\right)$ are blurry even though they have converged before the iteration limit $\left(\# R-R^{-1}=1800\right.$ and $\# R-R^{-1}=3600$ operations, respectively.). Similar to single- and double-helix scans, best results are obtained for when $P \leq 0.5$ and $N_{\theta} \geq 200$.

From these observations, for a given experiment acquisition time, where the amount of noise in the projections is proportional to the total number of projections:

i) Large $N_{\theta}$ is better to ensure reconstructions are not blurry (in a given iteration limit), but too large an $N_{\theta}$ provides noisy reconstructions. We have observed this threshold for this data at around $N_{\theta}=200$ which is half the Nyquist sampling criterion.

ii) A lower pitch always provides a better solution, assuming condition i) is satisfied. Although the lower the pitch, the noisier the projections, a lower pitch reduces noise effect into reconstructed slices since more projection values are averaged in the voxels. Using a double helix in order to correct for the non-uniform blur on 


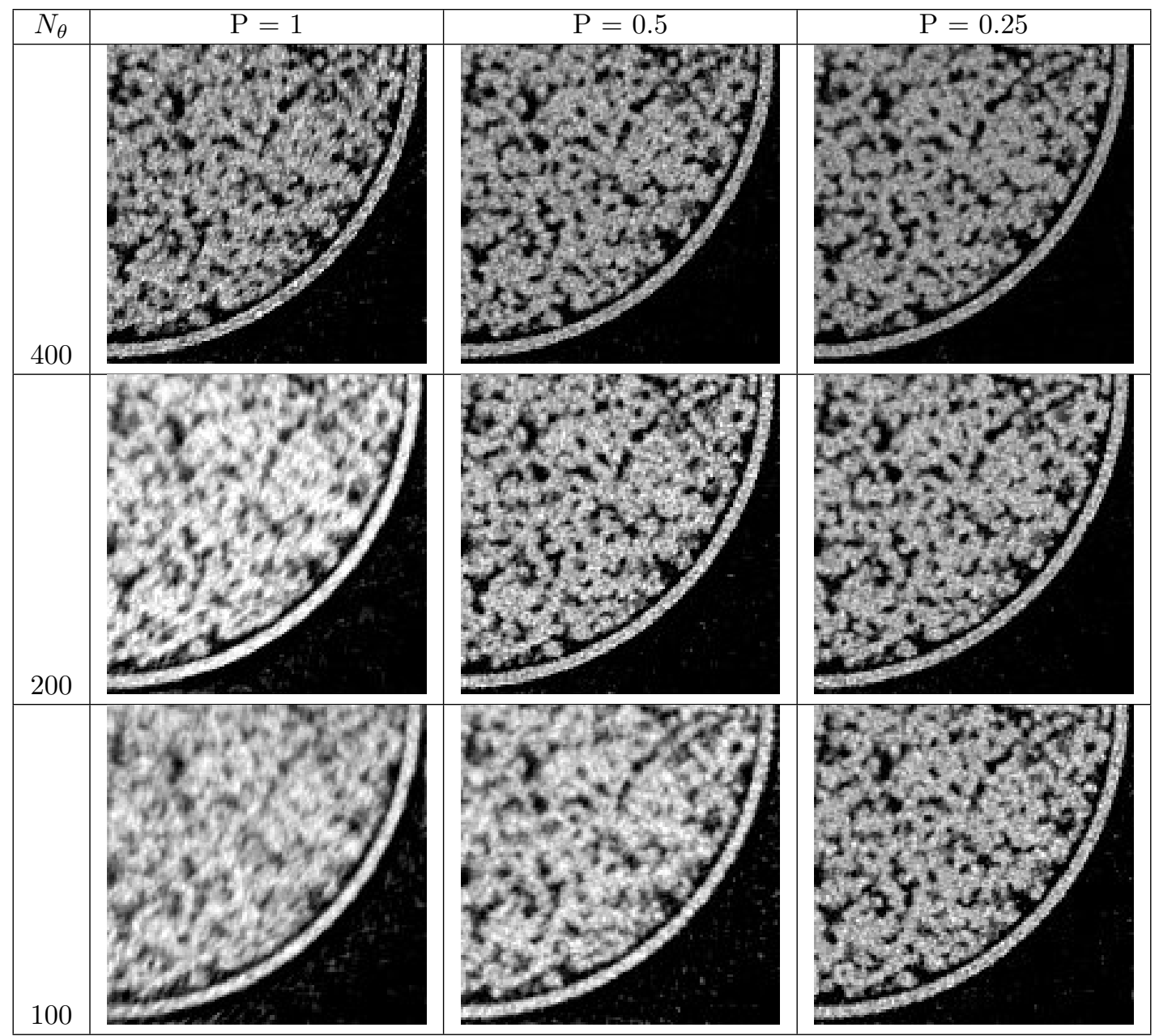

Figure 6. Result images of the central slice according to the parameters $P$ and $N_{\theta}$ for the single helix trajectory.

the reconstruction (due to the high-cone angle) is not relevant except when $P=1$, as illustrated on Fig. 10(a). $P<1$ provides a better homogeneity of the object in the viewing angles. As an illustration, note the similarity between the single-helix result $\left(N_{\theta}=200, P=0.5\right)$ and the double-helix result $\left(N_{\theta}=200, P=1\right)$, as depicted by the difference image on Fig. 10(b).

Now consider the XZ-slices of the reconstructed volume (see Fig. 11). According to the acquisition schemes, only the central 384 slices of the digital object (Fig.11) (a) can be reconstructed without distortion. However, using iterative reconstructions, an additional unwanted volume has to be reconstructed, in order to provide a "buffer" of the data backprojected out of the scan from one iteration to the next. This volume is delimited by the regions of the volumes (above and below the 384 central slices) in which backprojected data appear on Fig. 11(b-d). We note the efficiency of the adaptive trajectory compared to the others since a smaller amount of extra-volume has to be reconstructed. This reduced size leads to an improvement in: i) memory required to store the volume in RAM, and ii) iteration time since a smaller number of voxels require updating at each iteration. Furthermore, from the XZ-Slice Fig. 11(d), we cannot observe any distorsion in the central region of interest, which demonstrates the ease with which iterative reconstruction techniques can handle arbitrary trajectories.

\section{CONCLUSION}

In this paper we have assumed an iterative reconstruction technique (OSC) in order to enable a reduced number of projections and to utilise $100 \%$ of the acquired data in reconstruction. We have also set an experiment time 


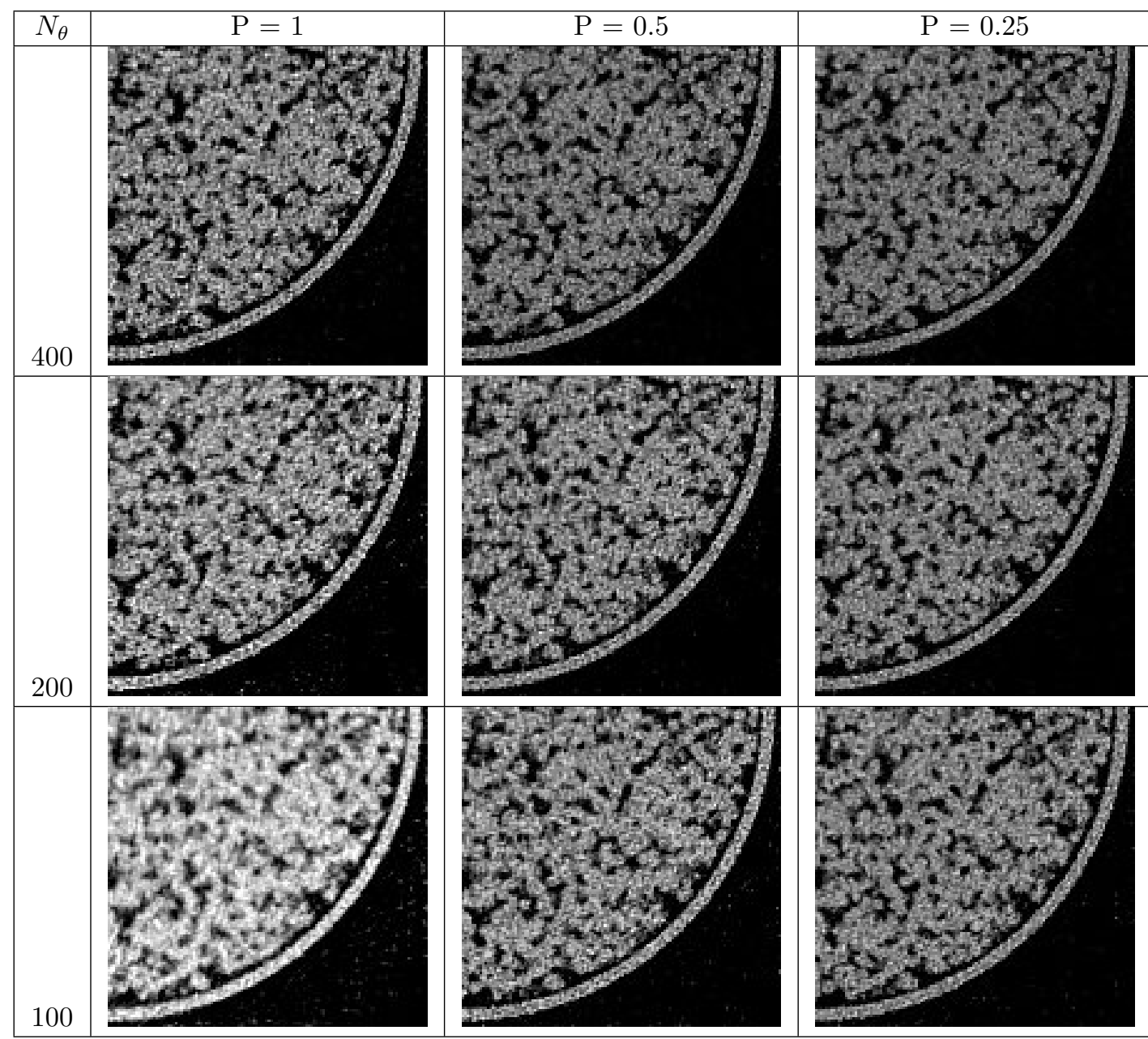

Figure 7. Result images of the central slice according to the parameters $P$ and $N_{\theta}$ for the double helix trajectory.

so that the dose was distributed amongst the number of projections required by the scanning protocol.

Given this we have found that adding more projections per revolution (up to Nyquist sampling) does improve resolution. However, in this case where reconstruction time was of consideration, convergence was obtained more quickly with less projections (200 per revolution) and produced comparable results to the unconverged datasets with a full complement of projections (400 per revolution). We also found that a lower pitch improves the reconstruction. Despite each projection being more noisy, there is less information wasted in overscan and more redundancy per voxel to result in a less-noisy reconstruction. A double-helix trajectory does correct for the non-uniform resolution introduced in high-magification, high-cone-angle imaging when the helical pitch is at a maximum (as specified by the Tam-Danielsson window). However, reducing the pitch by half (or more) with a single-helix similarly solves the problem. This solution has then benefit of reducing the span of the overscan and thus the additional unwanted volume required for iterative reconstruction. This in turn reduces memory requirements and speeds up each iteration in reconstruction. A helix with half circles replacing the overscan, here denoted the "adaptive trajectory," slightly reduces these requirements further.

This work has investigated experimental acquisition protocols. Future work involves investigating the most suitable reconstruction technique that will minimise reconstruction time while still producing results comparable to those using the Katsevich algorithm. 


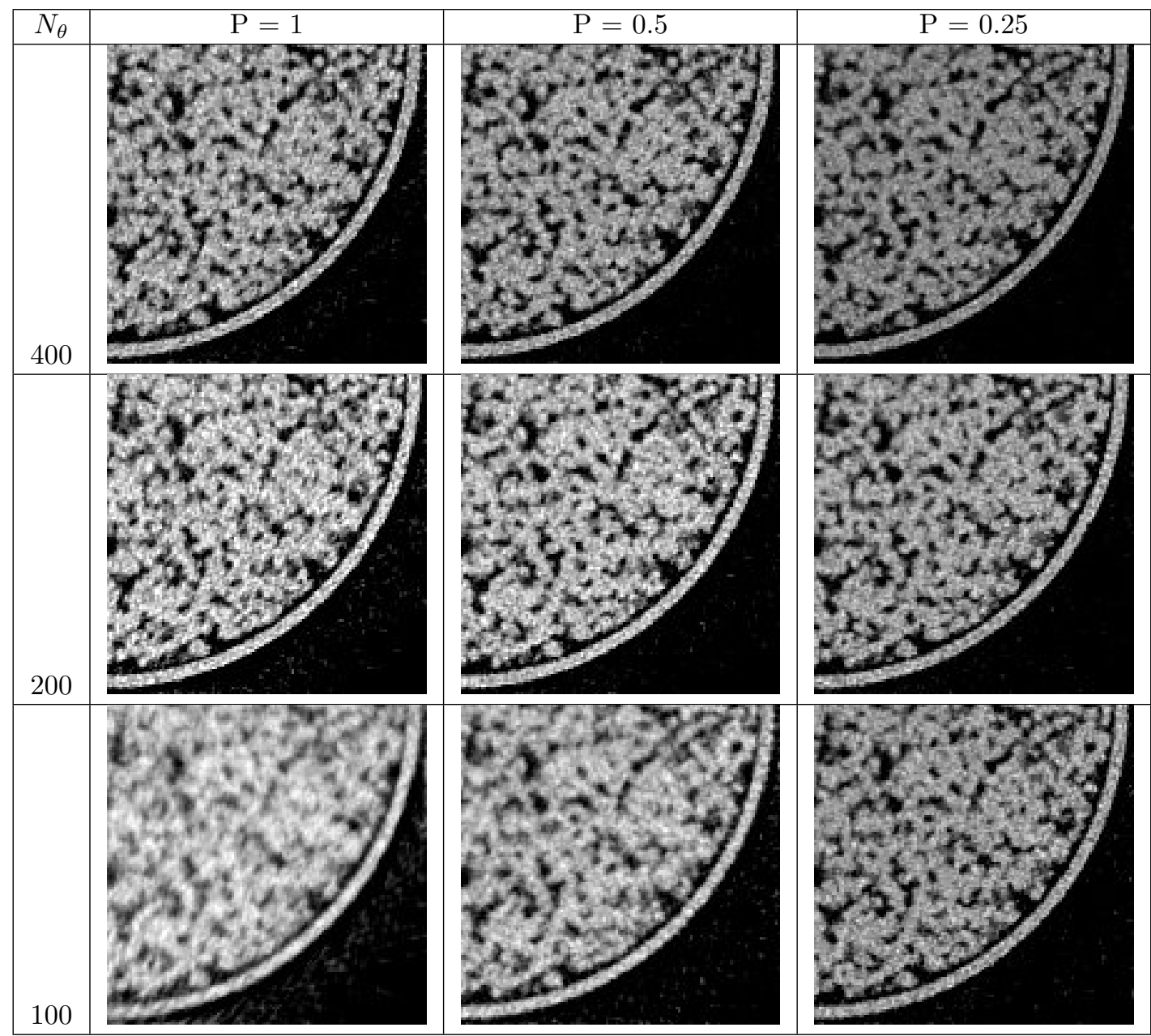

Figure 8. Result images of the central slice according to the parameters $P$ and $N_{\theta}$ for the adaptive trajectory.

\section{REFERENCES}

[1] Katsevich, A., "A general scheme for constructing inversion algorithms for cone beam ct," International Journal of Mathematics and Mathematical Sciences 2003(21), 1305-1321 (2003).

[2] Varslot, T., Kingston, A., Myers, G., and Sheppard, A., "High-resolution helical cone-beam micro-ct with theoretically-exact reconstruction from experimental data," Medical Physics 38(10), 5459-5476 (2011).

[3] Varslot, T., Kingston, A., Myers, G., and Sheppard, A., "Considerations for high-magnification high-coneangle helical micro-ct," In Proc. SPIE - International Society for Optical Engineering 8506, 8506.14 1-10 (2012).

[4] Gordon, R., Bender, R., and Herman, G. T., "Algebraic reconstruction techniques (art) for three-dimensional electron microscopy and x-ray photography," Journal of theoretical Biology 29(3), 471-481 (1970).

[5] Gordon, R., "A tutorial on art (algebraic reconstruction techniques)," Nuclear Science, IEEE Transactions on 21(3), 78-93 (1974).

[6] Andersen, A. and Kak, A. C., "Simultaneous algebraic reconstruction technique (sart): a superior implementation of the art algorithm," Ultrasonic imaging 6(1), 81-94 (1984).

[7] Shepp, L. A. and Vardi, Y., "Maximum likelihood reconstruction for emission tomography," Medical Imaging, IEEE Transactions on $\mathbf{1}(2), 113-122$ (1982).

[8] Lange, K. and Fessler, J. A., "Globally convergent algorithms for maximum a posteriori transmission tomography," Image Processing, IEEE Transactions on 4(10), 1430-1438 (1995). 


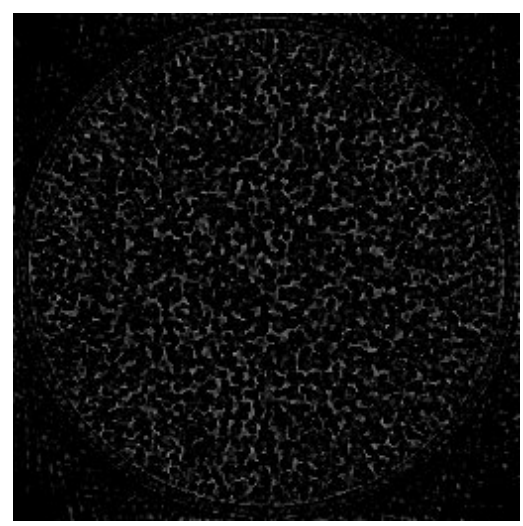

(a)

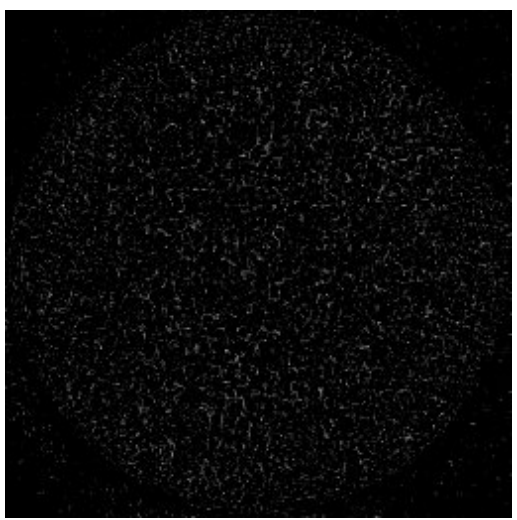

(b)

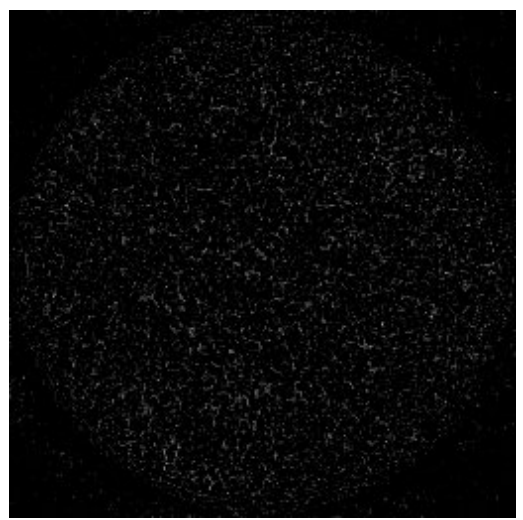

(c)

Figure 9. (a) Difference between single-helix $\left(N_{\theta}=100, P=0.5\right)$ and original slices highlights errors at the edges due to the low projection number. (b) Difference between single-helix $\left(N_{\theta}=200, P=0.5\right)$ and original slices shows a better accuracy at the edges. (c) Difference between single-helix $\left(N_{\theta}=400, P=0.5\right)$ and original slices highlights the better result one can achieve when Nyquist is reached, but with a few difference compared to $\left(N_{\theta}=200, P=0.5\right)$.

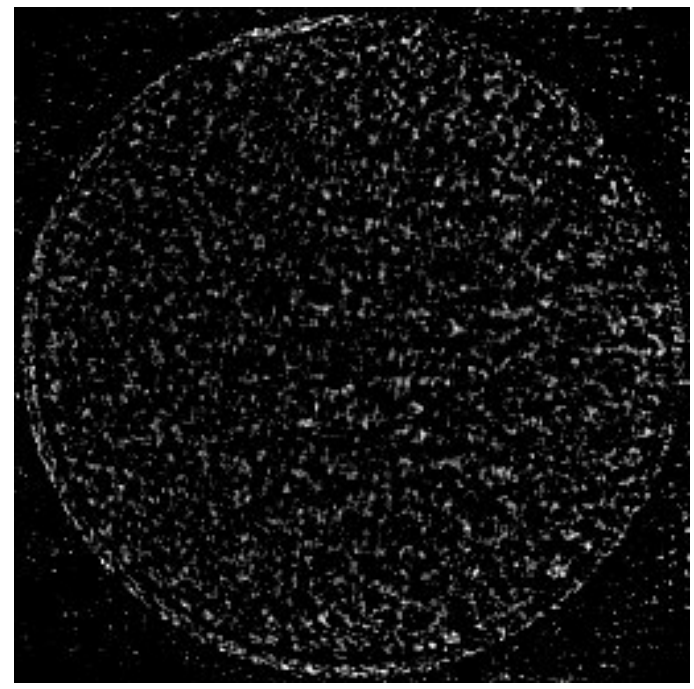

(a)

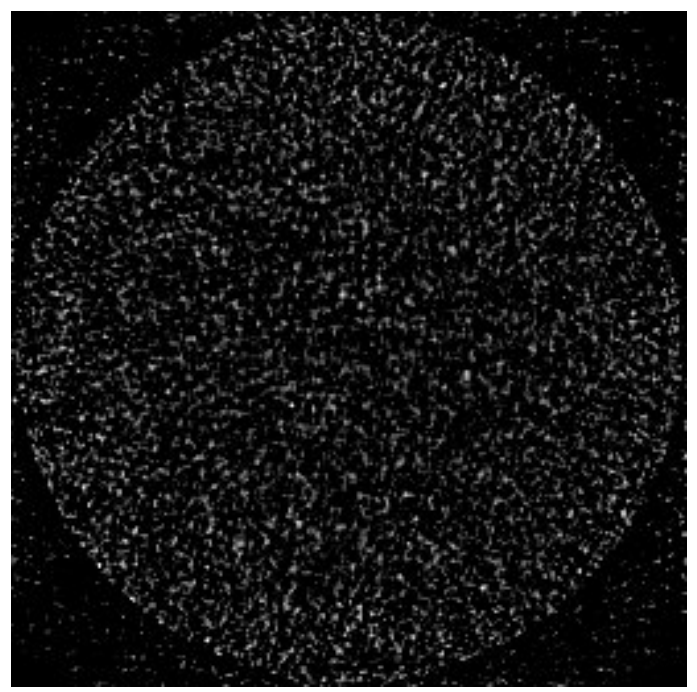

(b)

Figure 10. (a) Difference between single and double helix highlights the non-uniform blur effect (from left to right) appearing when using a single-helix. (d) Difference between single helix $\left(N_{\theta}=200, P=0.5\right)$ and double-helix $\left(N_{\theta}=\right.$ $200, P=1$ ) shows that just halving the pitch significantly reduces image distorsion due to non-uniform resolution.

[9] Erdogan, H. and Fessler, J. A., "Ordered subsets algorithms for transmission tomography," Physics in medicine and biology 44(11), 2835 (1999).

[10] Mayo, S., Miller, P., Gao, D., and Sheffield-Parker, J., "Software image alignment for x-ray microtomography with submicrometre resolution using a sem-based x-ray microscope," Journal of Microscopy 228, 257-263 (2007).

[11] A, S., Liu, X., and Salmon, P., "Compensation of mechanical inaccura- cies in micro-ct and nano-ct," In Proc. SPIE - International Society for Optical Engineering 7078, 7078.1C (2008).

[12] De Man, B., Nuyts, j., Dupont, P., Marchal, G., and Suetens, P., "An iterative maximum-likelihood polychromatic algorithm for ct," IEEE Trans. Medical Imaging 20(10), 999-1008 (2001).

[13] Recur, B., Paziresh, M., Myers, G., Kingston, A., Latham, S., and Sheppard, A., "Dual-energy iterative reconstruction for material characterisation," SPIE Optics and Photonics - accepted at Optical Engineering and Applications. (2014). 


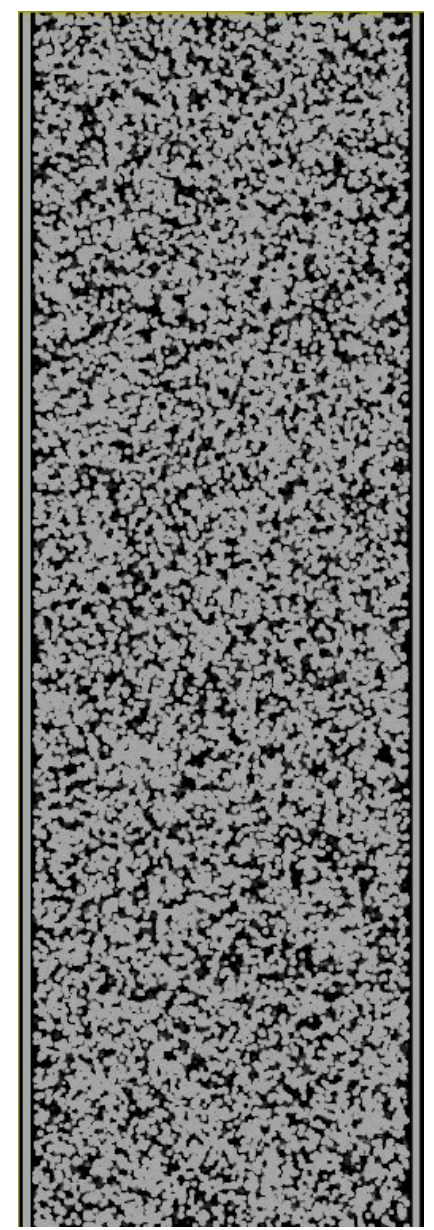

(a)

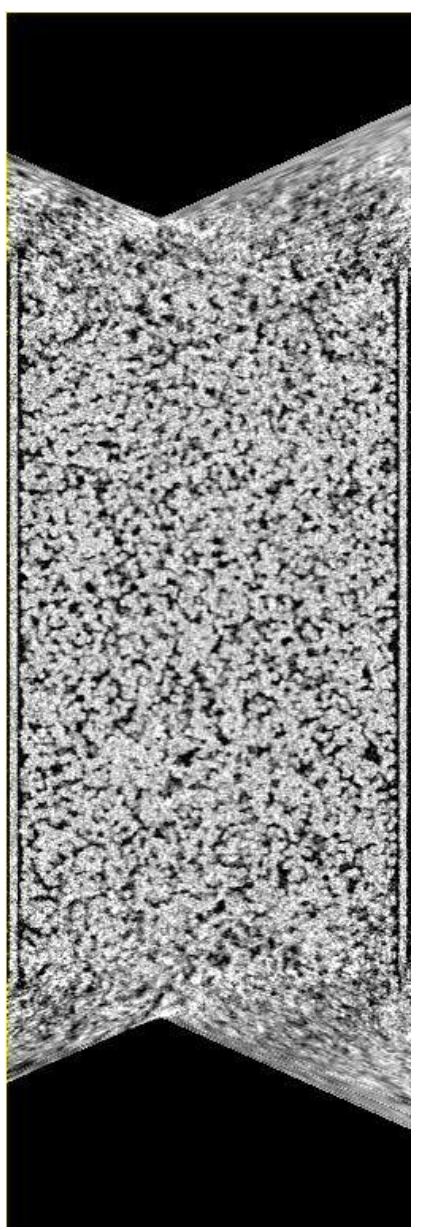

(b)

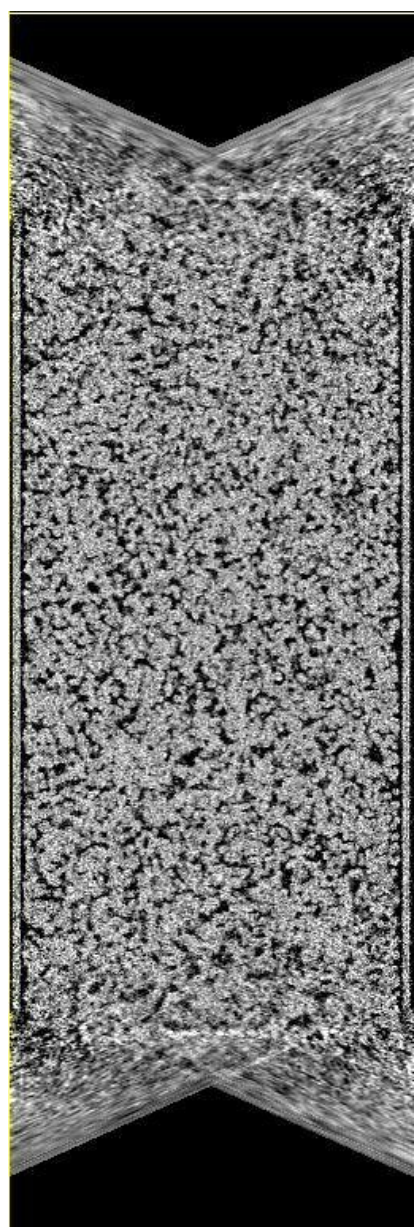

(c)

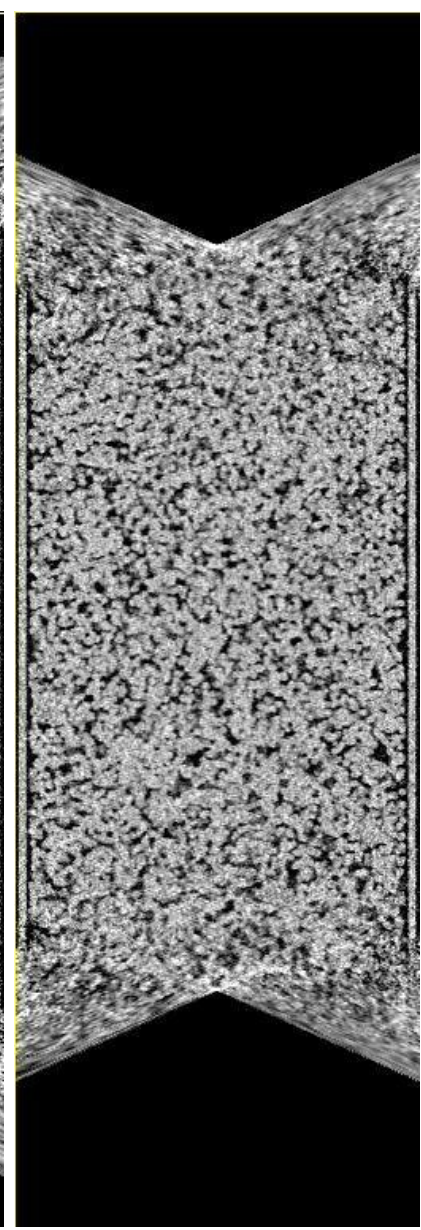

(d)

Figure 11. (a) Central XZ-Slice of the numerical object. Reconstructed XZ-Slices using: (b) single helix $\left(N_{\theta}=100\right.$, $N_{r}=5$, equiv. to 500 projections/scan), (c) double helix $\left(N_{\theta}=100, N_{r}=3\right.$, equiv. to 600 projections/scan) and adaptive trajectory $\left(N_{\theta}=100, N_{r}=5\right.$, equiv. to 500 projections/scan).

[14] Myers, G., "Improving dynamic tomography quality through a maximum a posteriori model," SPIE Optics and Photonics - accepted at Optical Engineering and Applications. (2014).

[15] Nuyts, J., De Man, B., Fessler, J. A., Zbijewski, W., and Beekman, F. J., "Modelling the physics in the iterative reconstruction for transmission computed tomography," Physics in medicine and biology 58(12), R63 (2013).

[16] Guan, H. and Gordon, R., "A projection access order for speedy convergence of art (algebraic reconstruction technique): a multilevel scheme for computed tomography," Physics in medicine and biology 39(11), 2005 (1994). 